\title{
Prognostic relevance of angiogenesis in stage III NSCLC receiving multimodality treatment
}

\author{
M. Kreuter*,\#,ף, M. Kropff* , A. Fischaleck*, K. Junker ${ }^{+}$, J. Gerss ${ }^{\S}$, A. Heinecke ${ }^{\S}$, \\ M. Lindermann ${ }^{\#}$, N. Reinmuth*,\#, W.E. Berdel ${ }^{\#}$, R.M. Mesters ${ }^{\#, f}$ and M. Thomas*,\#,f
}

ABSTRACT: Compelling evidence indicates that microvessel density (MVD) is a prognostic marker in early nonsmall cell lung cancer (NSCLC). However, its role in lymph node metastases in stage III NSCLC receiving multimodality treatment is unknown.

Lymph nodes of 142 patients with stage III NSCLC, treated in a trial of the German Lung Cancer Cooperative group, were evaluated for MVD. Median follow-up was 7.39 yrs. MVD was correlated with demographic and tumour-related variables and survival.

MVD (median 33.9) did not correlate with survival. However, in multimodality-treated stage IIIA patients receiving tumour resection with negative margins (R0), those with a high MVD had significantly prolonged overall survival with a median of 4.96 yrs compared with 1.99 yrs for those with low MVD $(p=0.041)$. Cox regression analysis revealed that MVD was a prognostic factor in R0-resected stage IIIA (hazard ratio 0.417). Furthermore, a significant correlation of MVD to stage was observed, with significantly lower MVD in stage IIIA than IIIB $(p=0.0062)$, and a significant correlation of MVD to histological subtype was observed, with adenocarcinoma revealing the highest scores $(p=0.0001)$.

Increased angiogenesis within lymph node metastases is a prognostic indicator for better survival in NSCLC patients. Thus, measurement of MVD might be useful in selecting patients for future neoadjuvant treatment decisions.

KEYWORDS: Angiogenesis, lymph node metastases, microvessel density, multimodality treatment, nonsmall cell lung cancer

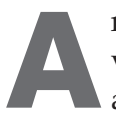

ngiogenesis, the formation of new microvessels from the pre-existing vasculature in a complex, multistep process, is involved in the growth, maintenance and metastasis of most solid tumours [1]. Several reports have demonstrated a significant correlation between neovascularisation assessed by intratumoural microvessel density (MVD) with clinicopathological factors and patient outcome in a variety of tumours [2].

Regarding the prognostic relevance of angiogenic activity in nonsmall cell lung cancer (NSCLC) as expressed by the intratumoural MVD, high MVD has been identified as a prognostic factor predictive of metastasis and poor survival [3]. Furthermore, high expression of vascular endothelial growth factor and other angiogenic growth factors within the primary tumour are considered as independent indicators of diminished outcome in NSCLC patients [4]. However, most of the studies have been conducted on early-stage lung cancer and little is known about the prognostic impact of angiogenesis in metastases, especially lymph node metastases, of NSCLC patients.

In locally advanced stages IIIA and IIIB of NSCLC with mediastinal lymph node metastases, locoregional treatment alone with surgery or radiotherapy results in disappointing long-term outcomes. Multimodality treatment approaches with pre-operative chemo(radio)therapy followed by surgery could improve outcome and enable surgery, even in stage IIIB disease [5, 6]. However, these treatment options are still under development [7]. The increasing availability of antibody
AFFILIATIONS

*Dept of Thoracic Oncology, Thoraxklinik, University of Heidelberg,

'Dept of Pneumology and Respiratory Critical Care Medicine, Thoraxklinik, University of Heidelberg, Heidelberg, \#Dept of Medicine, Haematology/ Oncology, University of Münster, ${ }^{\S}$ Dept of Medical Informatics and Biomathematics, University of Münster, Münster, +Institute of Pathology, Klinikum Bremen-Mitte, Bremen, Germany. ${ }^{f}$ Both contributed equally as senior authors.

\section{CORRESPONDENCE}

M. Kreuter

Dept of Pneumology and Respiratory Critical Care Medicine, Thoraxklinik, University of Heidelberg, Amalienstr 5, 69126 Heidelberg, Germany

Fax: 4962213961202

E-mail: michael.kreuter@ thoraxklinik-heidelberg.de

Received:

August 062008

Accepted after revision:

January 202009

\section{SUPPORT STATEMENT}

The clinical trial within which the current patients were treated was funded by grants from the German Cancer Aid, Bonn, Germany. It is registered at www.clinicaltrials.gov with reference number

NCT00176137

STATEMENT OF INTEREST

None declared. 
therapy and tyrosine kinase inhibitors raises interest in identifying patient subgroups that will benefit from additional systemic treatment, even in the multimodality approach. The inhibition of tumour angiogenesis has already been evaluated as a therapeutic strategy with great promise for the development of new lung cancer therapies. A wide range of different antiangiogenic drugs are currently under investigation or have already been licensed for the treatment of lung cancer [4].

In the present study, the clinical significance of neovascularisation in lymph node metastases in patients with locally advanced NSCLC before the initiation of a multimodal treatment strategy within a trial of the German Lung Cancer Cooperative Group (GLCCG) [8] was examined by correlating MVD with survival, response to therapy and further prognostic factors.

\section{MATERIALS AND METHODS \\ Patients and treatment}

Paraffin-embedded specimens of mediastinal lymph node biopsies from 142 patients with locally advanced, untreated stage III NSCLC were studied. Criteria for including patients in the present investigation were sufficient representative pretherapeutic biopsy material for MVD analysis and treatment within a randomised phase III trial of the GLCCG that was approved by the institutional review board and registered at www.clinicaltrials.gov (NCT00176137) [8]. Briefly, in the current protocol, patients with stage III NSCLC (invasive mediastinal staging obligatory) were stratified according to centre and stage (IIIA or IIIB) and then randomised to therapy. Therapy consisted in the experimental arm of three cycles of cisplatin $55 \mathrm{mg} \cdot \mathrm{m}^{-2}$ (days 1 and 4)/etoposide $100 \mathrm{mg} \cdot \mathrm{m}^{-2}$ (days $\left.1-4\right)(\mathrm{PE}$ ), followed by hyperfractionated radiotherapy (hfRT) (45 Gray; $2 \times 1.5 \mathrm{Gray} \cdot$ day $^{-1}$ ) with concurrent carboplatin $100 \mathrm{mg} \cdot \mathrm{m}^{-2} /$ vindesine $3 \mathrm{mg}$ (days 1, 8 and 15), then surgery. In inoperable patients or with positive (R1/2) resection, additional hfRT ( 24 Gray; $2 \times 1.5 \mathrm{Gray}$ day $^{-1}$ ) was offered. In the control arm patients were scheduled to receive three cycles PE, followed by surgery and then conventionally fractionated radiotherapy $\left(1.8 \mathrm{Gray}\right.$ day $\left.^{-1}\right)$ with 54 Gray or, if patients were inoperable or received R1/2 resection, with 68.4 Gray. Patients were excluded if written consent to the protocol or sufficient histological specimens were not available.

The following variables were evaluated for their distribution in the patient cohort and for possible correlations with outcome: patient age, sex, histological subtype, randomisation to treatment arm, stage, lymph node status at the time of diagnosis, resection margins of the primary tumour (negative (R0) versus positive (R1, R2)) and complete or incomplete resection. Complete resection was defined as R0 resection without metastastic involvement of the uppermost removed mediastinal lymph node.

\section{Immunhistochemical studies and evaluation of MVD}

Serial sections of paraffin-embedded biopsy specimens were processed for immunohistochemical identification of microvascular endothelial cells with an anti-CD31 antibody (clone JC/70A, working dilution 1:100; Dako, Hamburg, Germany). Immunohistochemical localisation was performed by the alkaline phosphatase/anti-alkaline phosphatase double bridge technique (Dako-APAAP kit; Dako). Before staining, tissue sections were deparaffinised in xylene, rehydrated in a graded ethanol series, and treated with proteinase $\mathrm{K}$ for antigen retrieval. The primary antibody was applied overnight at $4^{\circ} \mathrm{C}$. Subsequent steps were performed according to the manufacturer's instructions. The fast red substrate (Dako) was employed for revelation of phosphatase activity (10 min at room temperature). Sections were counterstained with $0.1 \%$ $(\mathrm{w} / \mathrm{v})$ haematoxylin.

The degree of angiogenesis was determined by the count of microvessels within three independent hot spots per section and in two sections, as described previously $[9,10]$. MVD was defined as the mean count of microvessels per $0.26-\mathrm{mm}^{2}$ field area (i.e. $\times 400$ field). The median interobserver and intersectional variability was low ( $2 \%$ and $4 \%$, respectively). The median MVD of the entire group was determined, in order to classify patients into two groups with high (>median) and low ( $\leqslant$ median) MVD according to an international consensus report [10].

\section{Statistical analysis}

The associations of (classified) MVD and categorical prognostic factors (i.e. sex, histological subtype, randomisation to treatment arm, stage, lymph node status, resection margins and resection status) were examined using Fisher's exact test. In order to examine the association of (classified) MVD and patient age, the U-test was applied.

The distributions of the time-to-event variables were estimated using the Kaplan-Meier method, and comparisons were based on the log-rank test. Median follow-up was estimated by means of the inverse Kaplan-Meier method. All potential prognostic factors assessed and MVD were tested using the Cox proportional hazards model.

All p-values reported are two-sided with a significance level of 0.05 . Statistical analyses were intended to be explorative and not confirmative. No adjustment for multiple testing was carried out. All calculations were performed using the SAS package (SAS Institute Inc., Cary, NC, USA).

\section{RESULTS}

\section{Patient characteristics}

The subjects of the present study were 142 patients with NSCLC treated within a phase III trial of the GLCCG [8]. There were 114 males and 28 females with a median (range) age of 59 (33-69) yrs. Patient characteristics are shown in table 1. 65 patients had squamous cell carcinoma, 62 had adenocarcinoma and 15 had large cell carcinoma. 77 patients were randomised to the experimental and 65 to the control arm. 59 patients were diagnosed with stage IIIA, 83 with stage IIIB. Surgery was performed on 88 of all patients with 73 patients receiving R0 resection and 51 patients with a complete resection. 38 of the patients with R0 resection had stage IIIA and 35 had stage IIIB disease.

\section{MVD in NSCLC}

The median microvessel count for all patients was 33.9 (per $\times 400,0.26-\mathrm{mm}^{2}$ field) with an interquartile range of 9.25-69.5. Patients were classified into two groups with high $(>33.9)$ and low ( $\leqslant 33.9)$ MVD, based on the median MVD of the entire group. Representative lymph node biopsy tissue specimens from lung cancer patients are shown in figure 1 for a tumour with low (fig. 1a) and a tumour with high (fig. 1b) MVD. 


\begin{tabular}{|c|c|c|c|c|}
\hline TABLE 1 & cteristics & & & \\
\hline & All patients & $\begin{array}{l}\text { High } \\
\text { MVD }^{\#}\end{array}$ & $\begin{array}{l}\text { Low } \\
\text { MVD" }\end{array}$ & $\mathrm{p}$-value \\
\hline \multicolumn{5}{|l|}{ Subjects } \\
\hline Age yrs & 59 (33-69) & 59 (33-69) & 59 (37-69) & 0.5468 \\
\hline \multicolumn{5}{|l|}{ Sex } \\
\hline Male & 114 & 51 & 63 & 0.0192 \\
\hline Female & 28 & 20 & 8 & \\
\hline \multicolumn{5}{|l|}{$\begin{array}{l}\text { Histological subtype of } \\
\text { NSCLC }\end{array}$} \\
\hline Squamous cell carcinoma & 65 & 21 & 44 & 0.0001 \\
\hline Adenocarcinoma & 62 & 43 & 19 & \\
\hline Large cell carcinoma & 15 & 7 & 8 & \\
\hline \multicolumn{5}{|l|}{ Stage } \\
\hline IIIA & 59 & 21 & 38 & 0.0062 \\
\hline IIIB & 83 & 50 & 33 & \\
\hline \multicolumn{5}{|l|}{ Randomised treatment arm } \\
\hline Experimental & 77 & 39 & 38 & 1.0000 \\
\hline Control & 65 & 32 & 33 & \\
\hline \multicolumn{5}{|l|}{ Surgery performed } \\
\hline Total & 88 & 40 & 48 & \\
\hline \multicolumn{5}{|l|}{ Tumour resection margins } \\
\hline Negative & 73 & 34 & 39 & 0.5734 \\
\hline Positive & 5 & 1 & 4 & \\
\hline Exploratory thoracotomy & 10 & 5 & 5 & \\
\hline \multicolumn{5}{|l|}{ Tumour resection $^{+}$} \\
\hline Complete & 51 & 27 & 24 & 0.1273 \\
\hline Incomplete & 22 & 7 & 15 & \\
\hline
\end{tabular}

Data are presented as $\mathrm{n}$ or median (range), unless otherwise stated. MVD: microvessel density; NSCLC: nonsmall cell lung cancer. \#: >median;

$\because: \leqslant$ median; ${ }^{+}$: in negative resection margins.

A significant correlation of MVD with stage was noticed $(p=0.0062)$. In stage IIIA, the median MVD was 22.4, while in stage IIIB the median MVD was 40.6. Concerning the nodal status, a high MVD correlated significantly with an advanced lymph node status (N3; $p=0.019)$. Furthermore, a statistically significant association could be detected between MVD and the histological subtype $(\mathrm{p}=0.0001)$. While $69.4 \%$ of patients with adenocarcinoma had a high MVD, this was only $33.2 \%$ in squamous cell carcinoma. Moreover, a statistically significant association between MVD and sex could be detected $(p=0.019)$. There was no statistically significant correlation between MVD and patient age, resection margins, resection status or treatment arm (data not shown).

\section{Correlation of MVD with overall and relapse-free survival}

The median (range) follow-up period was 7.39 (0.14-9.63) yrs. For all 142 patients analysed, overall survival (OS) and progression-free survival (PFS) did not differ between patients with high versus low MVD $(\mathrm{p}=0.80$ and $\mathrm{p}=0.87$, respectively; fig. $2 \mathrm{a}$ and $\mathrm{b}$ ). However, in stage IIIA patients receiving tumour $\mathrm{R} 0$ resection, OS was significantly prolonged in those with a high MVD, with a median of 4.96 yrs compared with those with a low MVD with a median survival of 1.99 yrs $(p=0.041)$. PFS was also prolonged (2.57 versus $1.62 \mathrm{yrs}$ ), but without reaching statistical significance $(p=0.089$; fig. $3 a$ and $b)$. To rule out the possibility that intercurrent disease might have interfered with the OS analysis, a mortality analysis was performed, which showed that most deaths were tumour related and did not alter the results of this analysis (data not shown).

The entire group of patients underwent further subgroup analysis according to lymph node status, treatment arm, resection margins and resection status. These factors were selected because they might impact outcome and interfere with the predictive impact of MVD [6, 8]. Subgroup analyses were performed upon the median MVD of the entire patient population (33.9) and upon the median MVD of the respective subgroups.

In the analysis of stage IIIA, the subgroup median MVD was 22.4. Here, with a median follow-up of 9.31 yrs, PFS was significantly prolonged in patients with a high MVD (median PFS $1.84 \mathrm{yrs}$ ) compared with a low MVD (median PFS $0.89 \mathrm{yrs} ; \mathrm{p}=0.05$ ) and
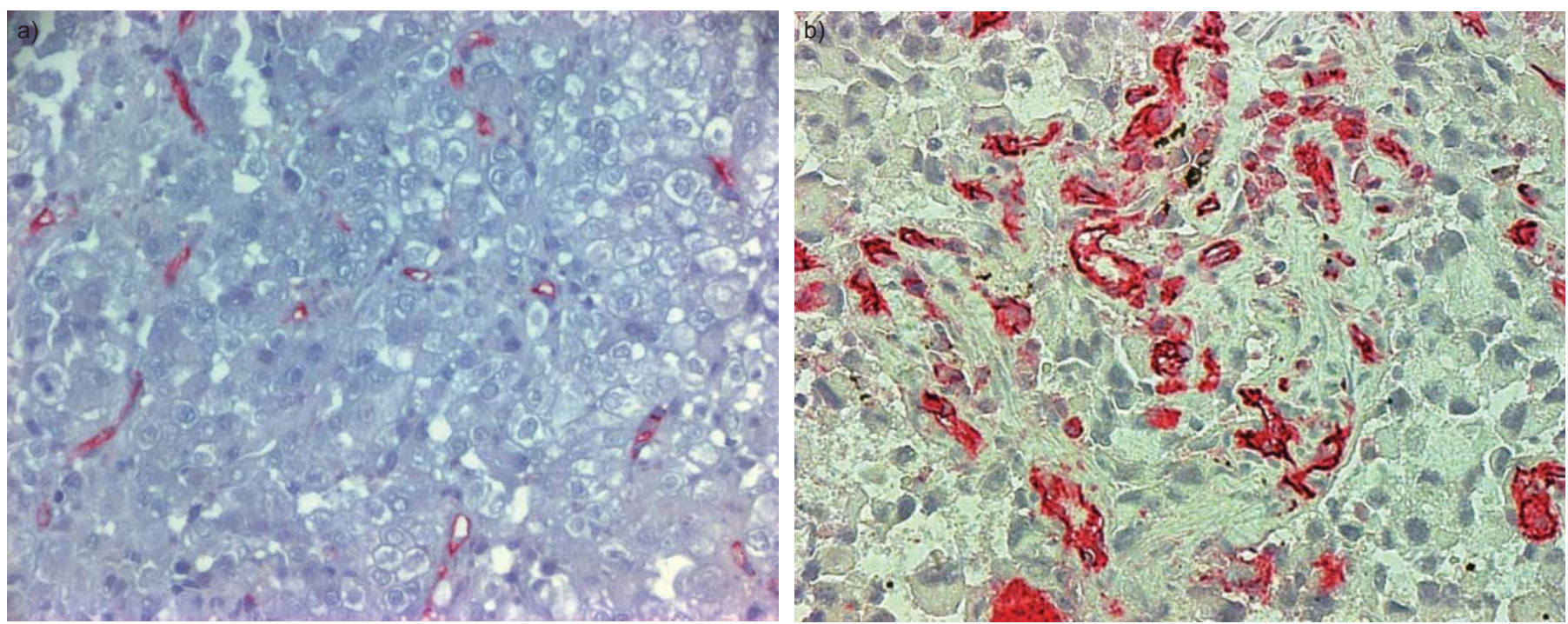

FIGURE 1. Immunohistochemical staining of nonsmall cell lung cancer lymph node metastasis tissue slides with anti-CD31 antibodies for the quantification of microvessel density (MVD). Sections from specimens with a) low and b) high MVD. Original magnification $\times 200$. 
for those IIIA patients with R0 resection $(p=0.03)$. OS showed a similar association in the stage IIIA subgroup (2.26 versus $1.62 \mathrm{yrs})$; however, without statistical significance $(\mathrm{p}=0.15)$.

For patients with stage IIIB, N2 or N3 lymph node status, treatment in the experimental or control arm, R0 resection or complete resection, OS and PFS did not differ with respect to high versus low MVD (data not shown).

Univariate Cox regression analysis identified MVD in patients with stage IIIA and R0 resection as an independent prognostic survival factor (hazard ratio $0.417, p=0.04$ ) and in the subgroup analysis for stage IIIA (cut-off 22.4) as a prognostic parameter for PFS (hazard ratio 0.580, $\mathrm{p}=0.05$ ). Furthermore, multivariate Cox regression analysis revealed that resection status (complete versus incomplete) was an independent prognostic factor for survival in the overall patient population $(\mathrm{p}=0.009)$.

\section{DISCUSSION}

The aim of the present study was to examine the clinical significance of neovascularisation in lymph node metastases of patients with stage III NSCLC prior to the initiation of polychemotherapy within the context of a multimodality treatment strategy. Despite progress in understanding the molecular biology of NSCLC its treatment remains challenging. Until now there has been a lack of clinical stratifying
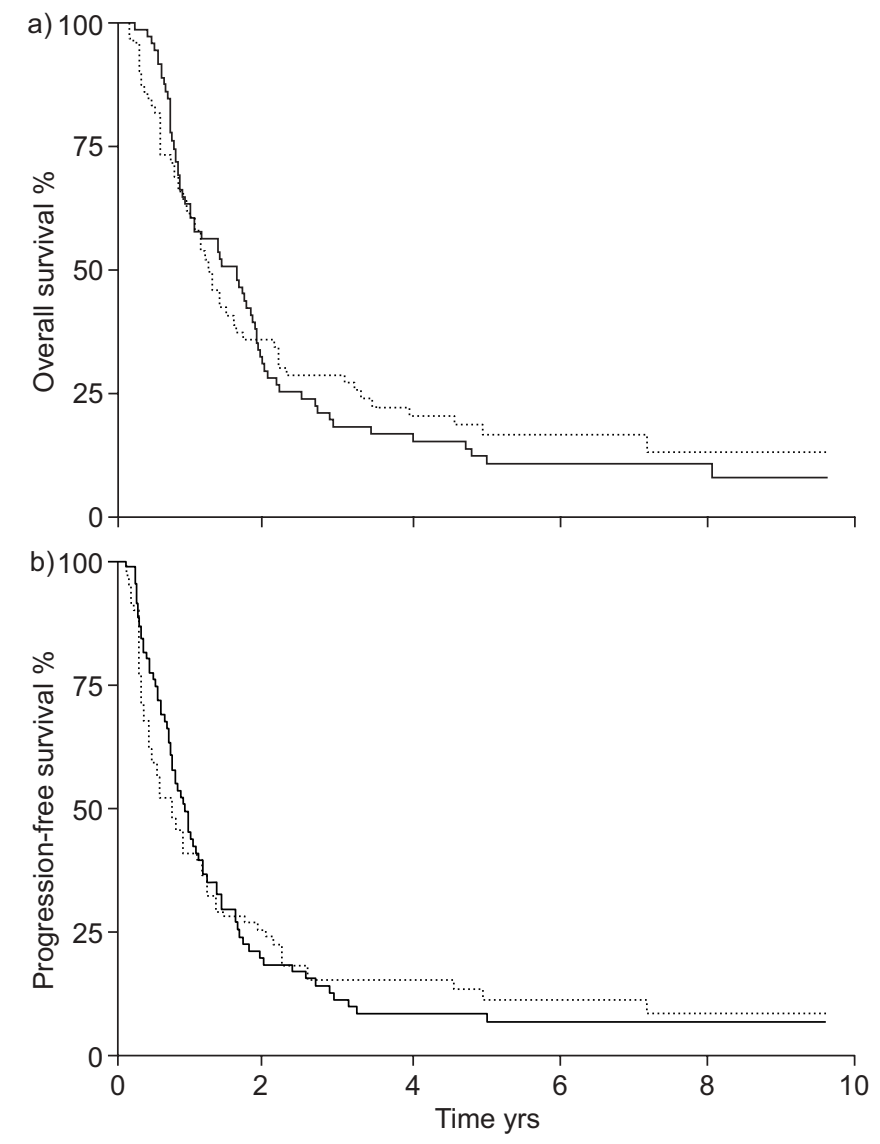

FIGURE 2. Kaplan-Meier estimates of a) overall and b) progression-free survival of all analysed patients ( $n=142)$. $\cdots \cdot$. microvessel density (MVD) $>33.9$; —: MVD $\leqslant 33.9$. Log ranks a) $p=0.80$ and b) $p=0.87$. categories before initiating chemotherapy in locally advanced NSCLC that would allow prediction of the response to therapy. Recent investigations have revealed that angiogenesis plays a significant role in the pathogenesis of NSCLC and in the mechanisms of disease progression [4].

The present study demonstrates for the first time in a large group of patients a significant association of MVD within mediastinal lymph node metastases with survival of stage IIIA NSCLC patients who received multimodality treatment including tumour R0 resection. Patients with a high degree of MVD within the metastases had a significantly enhanced survival compared with patients with low MVD.

The association of MVD and prognosis in NSCLC has already been reported by several others. The present data are in line with a report by CHANDRACHUD et al. [11], who showed that survival time was generally longer for NSCLC patients with higher vascularity. However, in contrast to the present results and those of CHANDRACHUD et al. [11], most other authors have described that high MVD is a poor prognostic factor for survival in NSCLC.
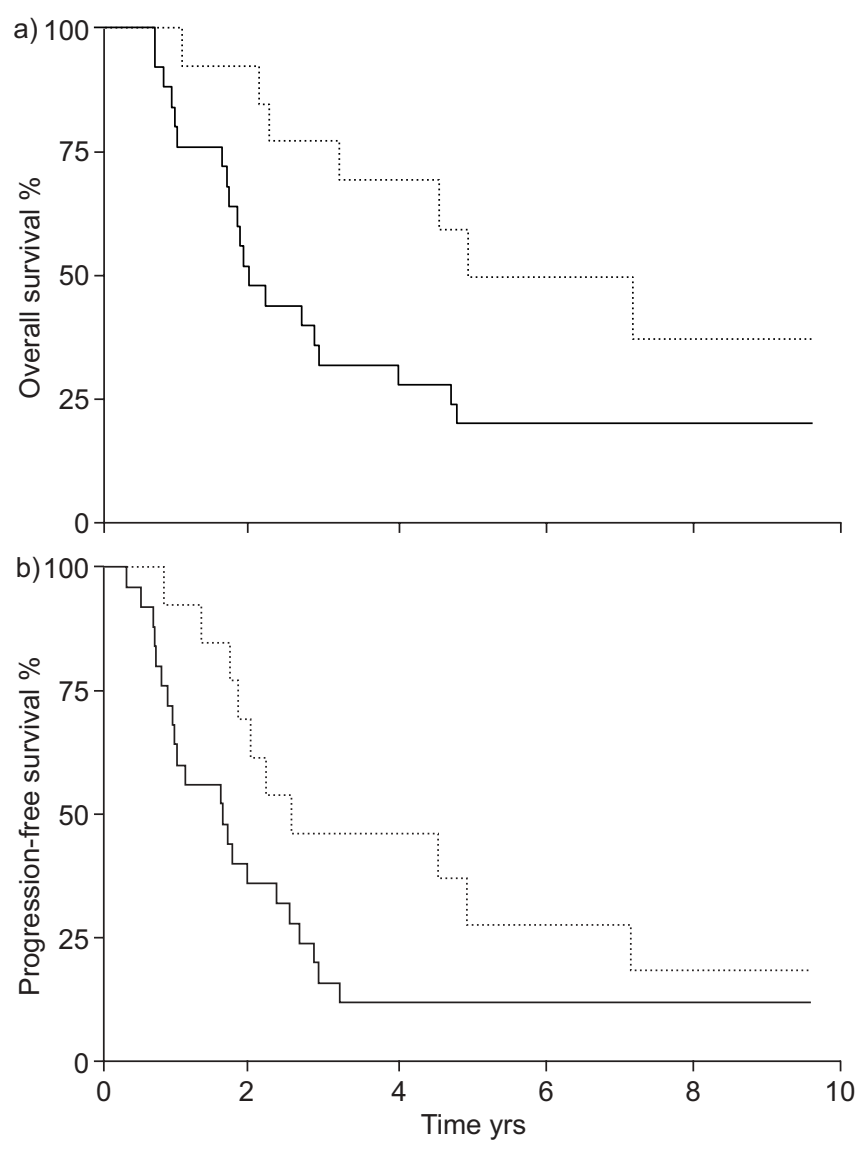

FIGURE 3. Kaplan-Meier estimates of a) overall and b) progression-free survival of stage IIIA nonsmall cell lung cancer patients with negative surgical resection margins grouped according to median microvessel density (MVD) of 33.9 $(n=38)$. a) Overall survival was significantly prolonged in patients with high MVD $(\cdots \cdots ;$ MVD >33.9) compared with patients with low MVD (-; MVD $\leqslant 33.9$; $p=0.041$. b) Progression-free survival was also prolonged but did not reach statistical significance $(p=0.089)$. 
Several factors have to be considered. First, most of these reports investigated MVD within primary tumours while the present study aimed to determine MVD within lymph node metastases. A study by ANGELETTI et al. [12] investigating a comparable population produced results contradictory to the present study, showing that a high MVD within the primary tumour was a negative prognostic factor. This might assume that comparing MVD between primary tumours and lymph node metastases is not reliable. Support of this hypothesis comes from a study by EDEL et al. [13], who demonstrated only varying associations between the level of angiogenesis in individual breast cancer tumours and their metastastic lymph nodes. Furthermore, GuIDI et al. [14] showed that only MVD within lymph node metastases, but not within primary mammary carcinomas, correlated with survival.

Secondly, most reports on the role of MVD in NSCLC determined angiogenesis in early stage lung cancer while the present study explored patients with locally advanced NSCLC. As prognosis differs remarkably within different stages of NSCLC, comparisons have to be made with caution.

However, the most important difference might be that other authors only investigated surgically treated patients while the present study patients were treated within a multimodality therapy concept. The integration of intensive polychemotherapy into a multimodality treatment strategy has led to distinct prognostic improvements in stage III NSCLC [5], which might be an explanation for better survival of patients with a high degree of MVD in the current report. As tumour microcirculation is an important factor in drug delivery to cancer cells [15], a higher density of blood vessels could hypothetically improve accessibility of the chemotherapy to proliferating lung carcinoma cells, i.e. the efficacy of drug delivery might be much higher in a tumour with a high degree of MVD than in a tumour with a low MVD. Support of this hypothesis comes from a study by VOLM et al. [16], who showed that MVD was significantly reduced in chemotherapy-resistant NSCLC tumours when compared with chemotherapy-sensitive tumours.

Additionally, direct anti-angiogenic mechanisms of cytotoxic chemotherapy by induction of endothelial cell apoptosis may contribute to the eradication of tumour cells. Indeed, direct toxic effects on endothelial cells as well as real anti-angiogenic effects have been described in in vitro and in vivo models for different cytostatic agents (e.g. anthracyclins, vinca alkaloids and paclitaxel) [17-20]. This hypothesis might also explain the difference between the results of ANGELETTI et al. [12] and those of the current study, which is most probably related to the different treatment modalities. In the current study all patients received intensive polychemotherapy, while only about a third of the patients in the report by ANGELETTI et al. [12] were treated chemotherapeutically. Furthermore, the effect of radiation or chemoradiotherapy has to be considered, as hypoxia within a tumour causes resistance to radiotherapy. Hypothetically, oxygenation might be improved within a highly vascularised tumour lesion, leading to enhanced response to therapy and thereby better survival. This hypothesis is supported by several reports that have already shown that higher MVD significantly correlates with better response and survival in solid tumours treated with chemoradiotherapy $[15,21]$.
Finally, our observation that MVD correlated with better survival in multimodality-treated stage IIIA patients with R0 resection but not in those with a complete resection is remarkable. A complete resection is defined as $\mathrm{R} 0$ resection without metastastic involvement of the uppermost removed mediastinal lymph node and is an indicator for enhanced survival in stage III NSCLC undergoing multimodality treatment [8]. Our finding might therefore give support to our assumption that lymph node metastases with an increased vascularisation have an improved response to chemo/radiotherapy compared with those with low MVD. Even if the uppermost mediastinal lymph node after R0 resection was involved, patients with a high MVD had an enhanced survival compared with those with a low MVD within the lymph node metastasis, which might be attributed to the effects of chemoradiotherapy.

Some of the discrepancies with the aforementioned reports may also be explained by the fact that details of the methodology used to assess MVD can influence its value as a prognostic indicator, e.g. the antibody (CD31, CD34 or von Willebrand factor) or whether MVD is assessed at the periphery or the centre of the tumour [22]. According to an international consensus, CD31 is the proposed standard for the quantification of intratumoural MVD [10]. However, it has to be discussed critically that estimation of MVD within lymph node metastases by CD31 might result in false positive results [23]. Nonetheless, our estimations of MVD within the lymph nodes were only performed in large metastatic lesions replacing the original lymph node structure.

It should be discussed critically why MVD was only a positive predictor of survival in stage IIIA. However, one has to consider that stage III NSCLC is a heterogeneous collection of patients and that survival in stage IIIB NSCLC is significantly diminished compared with stage IIIA. Furthermore, R0 resection could only be performed in a lower percentage of stage IIIB patients than stage IIIA patients, suggesting that the unfavourable resection status in stage IIIB patients influenced outcome the most. This hypothesis was supported by the finding that resection status was an independent prognostic factor for survival in the overall patient population analysed. Additionally, survival in locally advanced NSCLC depends on further aspects, such as comorbidity and other molecular and immunological factors. Nonetheless, we cannot rule out that our subgroup analysis resulted in a large increase in the type I error. However, our analyses are not of confirmatory level of evidence and we did not evaluate a pre-specified null hypothesis as the current analyses were explorative. Furthermore, in a large subset of the present analyses, a trend towards a better survival in patients with high MVD was found, although without reaching statistical significance.

Adenocarcinoma is the most common type of lung cancer in most recently reported series, and is the most frequent histological type in females and nonsmokers of either sex [24]. In regard to this, our observation that MVD correlated significantly with the histological subtype and that adenocarcinoma significantly more frequently had a high MVD is remarkable. Similar results were obtained by YUAN et al. [25], demonstrating significantly higher MVD for patients with adenocarcinoma than with squamous cell carcinoma. 
A further notable result of the current investigation was the correlation of MVD with stage and lymph node status. These observations are in line with others [25-27], underlining the important impact of angiogenesis in the progression of NSCLC and giving support for the reliability of our findings.

In conclusion, the present study provides evidence for the prognostic significance of MVD within lymph node metastases of stage IIIA NSCLC patients receiving R0 resection in a multimodality approach. Higher survival rates were demonstrated for patients with increased MVD. Thus, MVD might be a potentially useful predictive marker in patients treated within a multimodal treatment strategy and should be further explored as a potential tool for treatment stratification.

\section{ACKNOWLEDGEMENTS}

We are grateful to C. Sauerland (Dept of Medical Informatics and Biomathematics, University of Münster, Münster, Germany) for her excellent statistical advice, to M. Engels (Dept of Medicine, Haematology/Oncology, University of Münster) for her technical assistance and to N. Sellhast (Institute of Pathology, University of Bochum, Bochum, Germany) for her support.

\section{REFERENCES}

1 Folkman J, Watson $\mathrm{K}$, Ingber $\mathrm{D}$, et al. Induction of angiogenesis during the transition from hyperplasia to neoplasia. Nature 1989; 339: 58-61.

2 Hasan J, Byers R, Jayson GC. Intra-tumoural microvessel density in human solid tumours. Br J Cancer 2002; 86: 1566-1577.

3 Meert AP, Paesmans M, Martin B, et al. The role of microvessel density on the survival of patients with lung cancer: a systematic review of the literature with metaanalysis. Br J Cancer 2002; 87: 694-701.

4 Herbst RS, Onn A, Sandler A. Angiogenesis and lung cancer: prognostic and therapeutic implications. J Clin Oncol 2005; 23: 3243-3256.

5 Farray D, Mirkovic N, Albain KS. Multimodaltiy therapy for stage III non-small-cell lung cancer. J Clin Oncol 2005; 23: 3257-3269.

6 Thomas M, Hoffknecht P, Droege C, et al. Non-small-cell lung cancer: multimodality approach in stage-III resectable disease. Lung Cancer 2004; 45: Suppl. 2, S99-S105.

7 Van Meerbeeck JP. The controversial role of surgery in stage III NSCLC. Lancet Oncol 2008; 9: 607-608.

8 Thomas M, Rübe C, Hoffknecht P, et al. Effect of preoperative chemoradiation in addition to preoperative chemotherapy: a randomised trial in stage III non-smallcell lung cancer. Lancet Oncol 2008; 9: 636-648.

9 Kreuter M, Bieker R, Bielack SS, et al. Prognostic relevance of increased angiogenesis in osteosarcoma. Clin Cancer Res 2004; 10: 8531-8537.

10 Vermeulen PB, Gasparini G, Fox SB, et al. Quantification of angiogenesis in solid human tumours: an international consensus on the methodology and criteria of evaluation. Eur J Cancer 1996; 32: 2474-2484.

11 Chandrachud LM, Pendleton N, Chisholm DM, et al. Relationship between vascularity, age and survival in nonsmall-cell lung cancer. Br J Cancer 1997; 76: 1367-1375.
12 Angeletti CA, Lucchi M, Fontanini G, et al. Prognostic significance of tumoral angiogenesis in completely resected late stage lung carcinoma (stage IIIA-N2). Impact of adjuvant therapies in a subset of patients at high risk of recurrence. Cancer 1996; 78: 409-415.

13 Edel MJ, Harvey JM, Papadimitriou JM. Comparison of vascularity and angiogenesis in primary invasive mammary carcinomas and in their respective axillary lymph node metastases. Clin Exp Metastasis 2000; 18: 695-702.

14 Guidi AJ, Berry DA, Broadwater G, et al. Association of angiogenesis in lymph node metastases with outcome of breast cancer. J Natl Cancer Inst 2000; 92: 486-492.

15 Hironaka S, Hasebe T, Kamijo T, et al. Biopsy specimen microvessel density is a useful prognostic marker in patients with $\mathrm{T}(2-4) \mathrm{M}(0)$ esophageal cancer treated with chemoradiotherapy. Clin Cancer Res 2002; 8: 124-130.

16 Volm M, Koomägi R, Mattern J. Interrelationships between microvessel density, expression of VEGF and resistance to doxorubicin of non-small lung cell carcinoma. Anticancer Res 1996; 16: 213-217.

17 Belotti D, Vergani V, Drudis T, et al. The microtubuleaffecting drug paclitaxel has antiangiogenic activity. Clin Cancer Res 1996; 2: 1843-1849.

18 Bocci G, Nicolaou KC, Kerbel RS. Protracted low-dose effects on human endothelial cell proliferation and survival in vitro reveal a selective antiangiogenic window for various chemotherapeutic drugs. Cancer Res 2002; 62: 6938-6943.

19 Hill SA, Lonergan SJ, Denekamp J, et al. Vinca alkaloids: anti-vascular effects in a murine tumour. Eur J Cancer 1993; 29: 1320-1324.

20 Schirner M, Hoffmann J, Menrad A, et al. Antiangiogenic chemotherapeutic agents: characterization in comparison to their tumor growth inhibition in human renal cell carcinoma models. Clin Cancer Res 1998; 4: 1331-1336.

21 Zhang SC, Miyamoto S, Kamijo $T$, et al. Intratumor microvessel density in biopsy specimens predicts local response of hypopharyngeal cancer to radiotherapy. Jpn J Clin Oncol 2003; 33: 613-619.

22 Hlatky L, Hahnfeldt P, Folkman J. Clinical application of antiangiogenic therapy: microvessel density, what it does and doesn't tell us. J Natl Cancer Inst 2002; 94: 883-893.

23 Hattori $H$. Caution should be taken in using CD31 for distinguishing the vasculature of lymph nodes. J Clin Pathol 2003; 56: 638-639.

24 Thomas P, Rubinstein L. Cancer recurrence after resection: T1 N0 non-small cell lung cancer. Lung Cancer Study Group. Ann Thorac Surg 1990; 49: 242-247.

25 Yuan A, Yang PC, Yu CJ, et al. Tumor angiogenesis correlates with histologic type and metastasis in nonsmall-cell lung cancer. Am J Respir Crit Care Med 1995; 152: 2157-2162.

26 Giatromanolaki A, Koukourakis M, O’Byrne K, et al. Prognostic value of angiogenesis in operable non-small cell lung cancer. J Pathol 1996; 179: 80-88.

27 Fontanini G, Lucchi M, Vignati S, et al. Angiogenesis as a prognostic indicator of survival in non-small-cell lung carcinoma: a prospective study. J Natl Cancer Inst 1997; 89: 881-886. 